\title{
$A b$-initio and Statistical Calculations to Predict the Rate Constant of Several Ethers Reactions with Chloramine-T
}

\author{
Ahmed M. Sadoon ${ }^{1}$; Ayman Saeed Mohamed Taib ${ }^{2}$ \\ ${ }^{1,2}$ Department of Chemistry, College of Education for Pure Science, University of Mosul, Mosul \\ Email: ${ }^{1}$ ams95@uomosul.edu.iq, ${ }^{2}$ ayman535@uomosul.edu.iq \\ (Received October 27, 2021; Accepted November 27, 2021; Available online 01 December 2021)
}

DOI: 10.33899/edusj.2021.126884.1196, (C) 2021, College of Education for Pure Science, University of Mosul.

This is an open access article under the CC BY 4.0 license (http://creativecommons.org/licenses/by/4.0/).

\begin{abstract}
.
The prediction of rate constant of Ethers reaction with Chloramine-T has been studied theoretically in this work using ab-initio and statistical calculations. The first order reaction between Ethers and Chloramine-T shows that the rate constant depends on the Ethers concentration. Therefore, ab initio calculations were used using DFT/ b3lyp with 6-311++G** as a basis set to extract the most stable geometry of the chosen ethers. Also, several physical parameters have been gained form these calculations such as mulliken charge at the active atoms, dipole moment, Homo and Lomo energy levels, Hardness, Electronic Chemical Potential and Global Electrophilicity Index. The relationship between these physical variables plays an effective role in the reaction rate. Therefore, statistical calculations were used to investigate the possible relationship between these physical parameters. These relationships can be predicted from the value of the correlation coefficient (R2) and the Standard error (SE). Simple regression analysis and multiple regression analysis were performed between these physical variables as independent variables and rate constant as a dependent variable using SPSS software. The results show high value of R2 (0.993) of the regression analysis which reflects the high relationship between these variables that affects the reaction rate concentration. The out data illustrates negligible differences (about 0.008) between the experimental and calculated rate constant value.
\end{abstract}

Keywords: rate of constant, $a b$ initio, Ethers, Chloramine-T.

$$
\begin{aligned}
& \text { استخدام حسابات ab-initio والاحصائية لتعيين ثابت سرعة التفاعل لمجموعة من الايثرات مع الكلورامين } \\
& \text { احمد موفق سعدون و أيمن سعيد محمد طيب } \\
& \text { قسم الكيمياء، كلية التربية للعلوم الصرفة، جامعة الموصل، الدوصل، العراق }
\end{aligned}
$$

$$
\begin{aligned}
& \text { تم دراسة معدل ثابت سرعة التفاعل للإيثرات مع الكلورامين - T نظريًا في هذا العمل باستخدام حسابات ab-initio والحسابات } \\
& \text { الإحصائية. يظهر تفاعل الدرجة الأولى بين الايثرات و Chloramine-T أن ثابت معدل سرعة التفاعل يعتمد على تركيز الإيثرات. }
\end{aligned}
$$

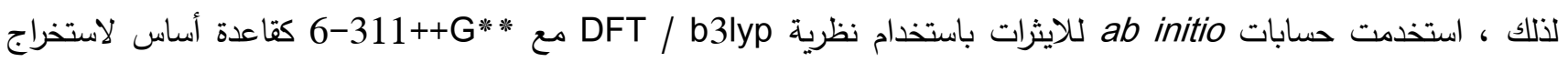

$$
\begin{aligned}
& \text { الهيكل الفراغي المستقر للايثرات قيد الدراسة. كذلك استخلصت قيم العديد من الثوابت الفيزيائية مثل شحنة موليكان للذرات في مركز }
\end{aligned}
$$


التفاعل ، والعزم ثنائي القطب و مستويات الطاقة Homo و Lomo و الصلابة والجهد الاكتروني الكيميائي واخيرا مؤشر الالكتروفيلية العالمية. تلعب العلاقة بين هذه المتغيرات الفيزيائية دورًا فعالًا في معدل سرعة تفاعل. لذلك ، تم استخدام الحسابات الإحصائية لفحص العلاقة المحتملة بين هذه الثوابت الفيزيائية. يمكن العثور على هذه العلاقات من خلال قيمة معامل الارتباط (R2) والخطأ القياسي (SE). درس تحليل الانحدار البسيط وتحليل الانحدار المتعدد بين هذه المتغيرات الفيزيائية كمتغيرات مستقلة وثابت معدل سرعة التفاعل كمتغير تابع باستخدام برنامج SPSS حيث أظهرت النتائج ارتفاع قيمة RS بما يعادل (0.993) لتحليل الانحدار المتعدد مما يعكس العلاقة العالية بين هذه المتغيرات التي تؤثر بثكل كبير على معدل سرعة تفاعل. كذلك أظهرت النتائج فروقا طفيفة (حوالي 0.008) في قيمة معدل ثابت سرعة التفاعل العملية والنظرية مما يظهر فعالية هذه الطريقة في ايجاد قيمة ثابت التفاعل الايثرات مع الكلورامين T- Tالطرق النظرية.

الكلمات المفتاحية: ثابت سرعة التفاعل, الايثرات, Chloramine-T ,ab-initio

المقدمة.

لعبت الحسابات النظرية دورًا مهما في الكثير من الدراسات الحديثة لتقدير العديد من الثوابت الكيميائية المختلفة مثل التنبؤ بثابت سرعة التفاعل عن طريق حسابات نظرية الاوربتال الجزيئي وحسابات الحالة الانتقالية [1] [2] والعديد من الدراسات الأخرى التي لتئي اهتمت بدراسة معدل ثابت سرعة التفاعل.[6-3. في هذا البحث, تم الجمع بين حسابات ab initio والحسابات الإحصائية للتنبؤ بمعدل ثابت سرعة التفاعل. الفكرة العامة من هذه الدراسة هي استخدام هذه الحسابات للعثور على العلاقة المؤثرة لبعض المتغيرات الفيزيائية على معدل ثابت سرعة التفاعل ثم إيجاد وتقدير معدل ثابت من خلال الحسابات الإحصائية. باستخدام هذه الحسابات ، يمكن بسهولة استخراج معدل التفاعل نظريا للتفاعلات الأخرى التي يصعب إيجاد ثابت سرعة التفاعل لها تجريبًا.

تعتبر الايثرات من المركبات ذات الاستخدام الواسع في الصناعة حيث تُستخدم أبخرة بعض الإيثرات كمبيدات حشرية مثل

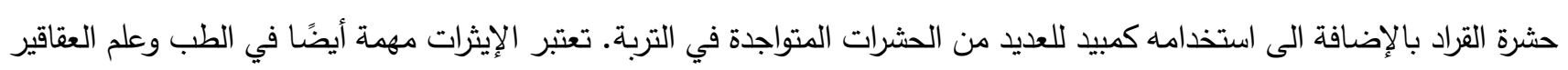
خاصة في التخدير حيث استخدمت Diethyl ether ، المعروف ببساطة باسم الأثير ، لأول مرة كمخد جراحي في عام 1842. هذا بالإضافة الى العديد من الاستخدامات المعروفة[7].

بثكل أساس تم اختيار تفاعل الايثرات مع Chloramine-T لاستخراج ثابت المعدل نظريًا. تم حساب ثابت السرعة لهذا التفاعل تجريبًا [8] وظهر أنه تفاعل من الرتبة الأولى. هذا يعني أن معدل ثابت السرعة يعتمد على مركب واحد وهو الايثرات. من المعلوم ايضا أن معدل ثابت السرعة يعتد على عدة عوامل فيزيائية. لذلك ، فإن الخطوة الأولى في الحسابات هي استخراج هذه فئه الثوابت الفيزيائية لمركبات الايثرات قيد الدراسة باستخدام حسابات ab-initio عن طريق إيجاد التركيب الكيميائي الأكثر استقرار لهذه الايثرات بعد ذلك تستخرج قيم المتغيرات الفيزيائية من هذه التراكيب الكيميائية. المتغيرات الفيزيائية التي تمت دراستها في كثير من الدراسات النظرية هي شحنة موليكان mulliken charge على الذرات النشطة [9]، الصلابة Hardness (ך) [10]، الجهد الاكتروني

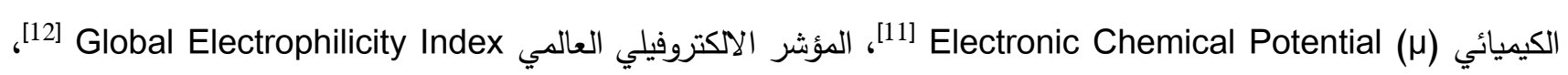
عزم ثنائي القطب (DM) dipole moment) واخيرا مستويات طاقة الهومو والومو [13]. 
الخطوة الرئيسة في هذه الدراسة هي إيجاد العلاقة بين هذه المتغيرات الفيزيائية التي تلعب دورًا فعالًا في معدل ثابت السرعة للتفاعل باستخدام الحسابات الإحصائية. يمكن إيجاد هذه العلاقات من خلال التحقق في قيمة معامل الارتباط correlation (R2) coefficient وتحليل الانحدار المتعدد بين المتغيرات الفيزيائية واعتبارها متغيرات مستقلة وبين معدل ثابت سرعة التفاعل كمتغير تابع.

\section{الجزء العملي}

ab-initio 1

تم إجراء الحسابات لمركبات الايثرات باستخدام حزمة برامج ORCA [14] وباستخدام نظرية (DFT/ B3LYP) والقاعدة الاساسية (311++G-6) للحصول على الأشكال الهندية للايثرات الأكثر استقرارا اخذين بالاعتبار إمكانية وجود العديد من الايزومرات وإيجاد الايزومر ذو الطاقة الاوطا لكل مركب من المركبات المختارة. تم دراسة تاثير المذيب (الماء) على هذه الاشكال الهندسية ايضا في هذه الحسابات بالإضافة الى تعيين درجة الحرارة لكل مركب مثل ما تم حسابه عمليا. في البداية, اختبرت العديد من التراكيب الفراغية الأولية المختلفة في محاولة للعثور على أكبر عدد ممكن من ألايزومرات. تم إيجاد التراكيب الفراغية أولاً باستخدام نظرية Hartree-Fock (HF) لتقليل الوقت المستغرق للحساب. بعد ذلك تم إعادة حساب طاقة التراكيب باستخدام نظرية DFT لزيادة دقة النتائج المستخرجة نظرا لدقة نظرية DFT العالية مقارنة بنظرية HF [15]. أخيرًا، استخرجت المتغيرات الفيزيائية من بيانات التراكيب النهائية ذات الطاقة الاوطا.

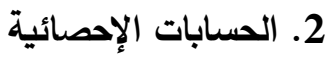

أجريت الحسابات الإحصائية باستخدام برنامج 2016 SPS [16]. تم تطبيق ارتباطات الانحدار الفردية والمتعدة بين المتغير الفيزيائي للايثرات كمتغير مستقل وثابت معدل التفاعل المستخرج عمليا لهذه الايثرات كمتغير تابع. تم اختيار المتغيرات الفيزيائية التي لها قيم جيدة لـ R2 و SE لتقدير المعدل النظري للثابت باستخدام معادلة الانحدار الخطي ، الدعادلة (1).

$$
\mathbf{Y}=\mathbf{a X}+\mathbf{b}
$$

= المتغير المستقل (المتغيرات الفيزيائية) S متغير تابع (القيمة التجريبية لمعدل ثابت سرعة التفاعل) X = ثابت (من الحسابات الإحصائية) ثابت (من الحسابات الإحصائية)

ولحسابات تحليل الانحدار المتعدد ، تم استخدام معادلة الانحدار الخطي والمبية ادناه في المعادلة رقم (2). $\mathbf{Y}=\mathbf{a} 0+\mathbf{a}_{1} \mathbf{x}_{1}+\mathbf{a}_{2} \mathbf{X}_{2}+\mathbf{a}_{3} \mathbf{X}_{3}+\mathbf{a}_{4} \mathbf{X}_{4}+\mathbf{e t c}$ 
نظرًا لأن معدل سرعة التفاعل تتأثر بشكل كبير بمجموعة R في تركيب الكيميائي للايثر الذي يكون بالصيغة التالية التالية (R-(C=O)-R) ومدى اختلاف قيم ثوابت سرع التفاعل بين هذه الايثرات.

أظهرت حسابات ab-initio باستخدام نظرية DFT/ b3lyp مع مجموعة الأساس (a)+G-311+G) العديد من التراكيب

الفراغية للايثرات قيد الدراسة وتم ايجاد التركيب الفراغي لكل مركب الذي يحمل اوطأ طاقة وكما هو مبين في الجدول (1). جدول (1): التراكيب الفراغية للايثرات ذات الطاقة الاوطأ وإلمحسوبة نظريا باستخدام *b3lyp/6-311++G*

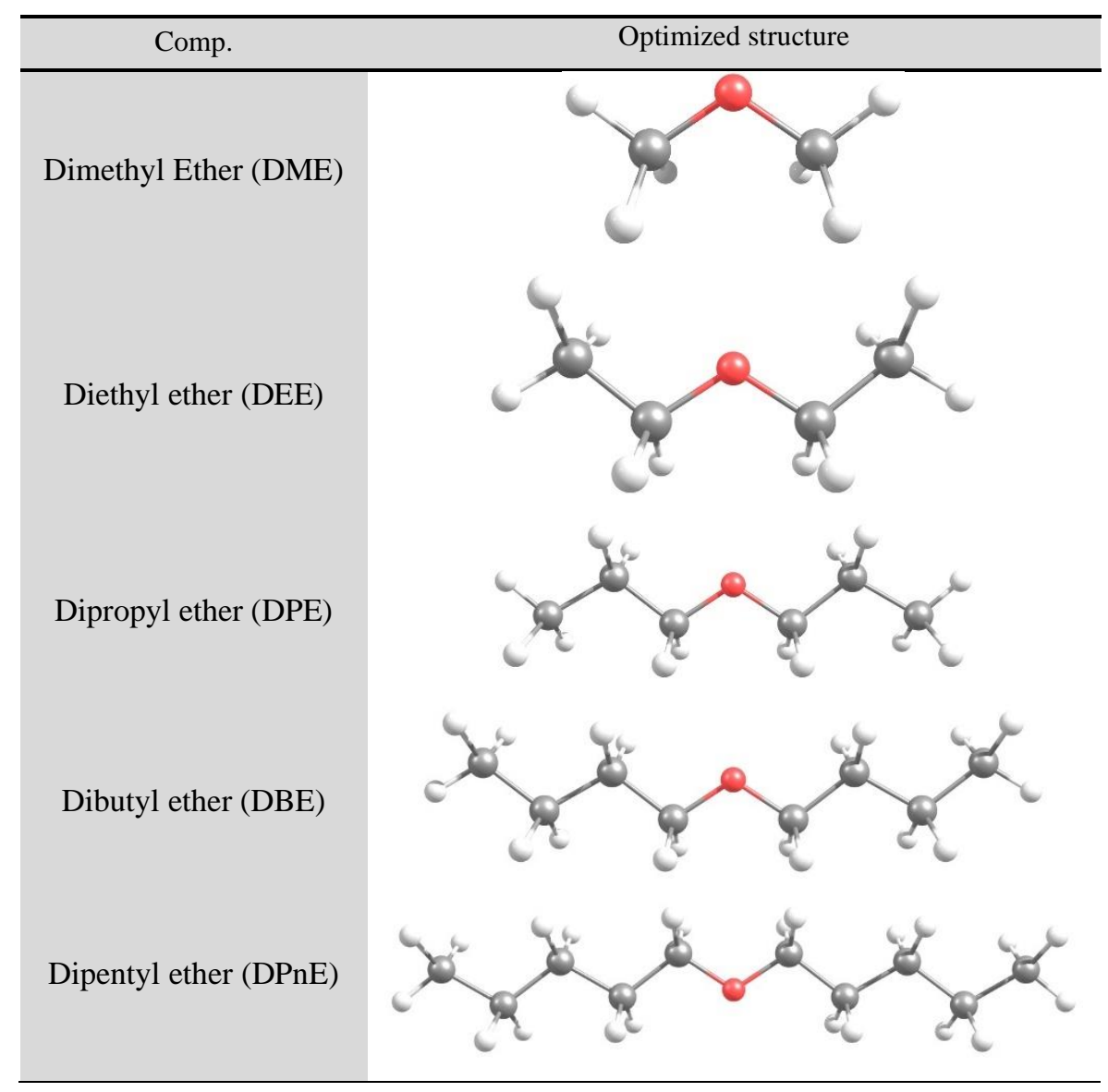

تظهر الدراسة ان الذرات النشطة للايثرات تكون في موقع التفاعل الذي يتكون من ذرة الاوكسجين وذرتي الكاربون المرتبطة بذرة الاوكسجين مباشرة بالاضافة الى ذرتي الهيدروجين المرتبطتان بذرتي الكاربون وتكونان في نفس مستوى ذرة الاوكسجين وتقعان تحت تاثير الثحنة السالبة له[11]. تم ترقيم هذه الذرات بالثكل الاتي (O1, C2, C4, H3, H5) وكما موضح في الثكل (1). تم اختيار هذه الذرات ايضا وفقًا للتغير الكبير في شحنتها مع ازدياد طول السلسلة المعوضة. 


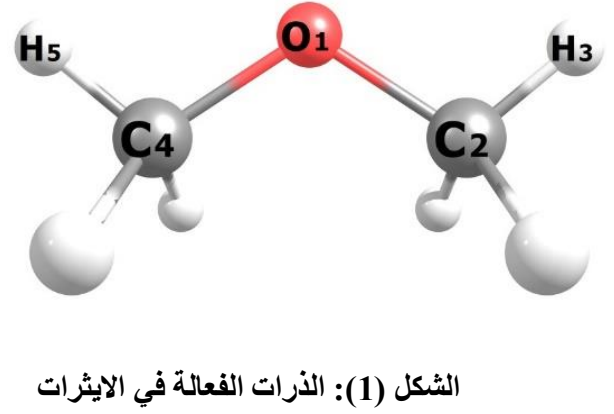

الجدول رقم (2) يوضح التغير في شحنة هذه الذرات لجميع الايثرات قيد الدراسة حيث يلاحظ انخفاض شحنة الذرات الفعالة تدريجيا مع ازدياد طول السلسلة المعوضة. الأساس النظري الذي سيتم اعتماده في هذه المقارنة هو مدى الاختلافات في توزيع الثحنات على الذرات في مركز التفاعل حيث أن الاختلاف الكبير في توزيع الثحنات على الذرات في مركز التفاعل يمثل حساسية الطريقة المعتمدة في هذا النوع من التطبيقات [18]. أيضًا ، تعتمد قيم الثحنات على الذرات قيد البحث على قدرة مجموعات R على إطلاق أو سحب الإكترون من مركز التفاعل [19].

الجدول (2): قيم شحنة الذرات الفعالة للايثرات المحسوبة نظريا باستخذام نظرية *3lyp/6-311++G

\begin{tabular}{|c|c|c|c|c|c|c|}
\hline \multirow{2}{*}{ Comp. } & \multicolumn{5}{|c|}{ Charge on atom (AU unit) } & \multirow{2}{*}{$\begin{array}{c}\text { dipole } \\
\text { moment } \\
\text { (C.m unit })\end{array}$} \\
\hline & 01 & C2 & H3 & $\mathrm{C4}$ & H5 & \\
\hline (DME) & -0.2150 & -0.2611 & 0.1215 & -0.2823 & 0.1214 & 1.4436 \\
\hline (DEE) & -0.1462 & -0.2611 & 0.1260 & -0.0730 & 0.1503 & 1.2221 \\
\hline (DPE) & -0.1183 & -0.2103 & 0.1232 & -0.1212 & 0.1443 & 1.0182 \\
\hline (DBE) & -0.1056 & -0.1951 & 0.1285 & -0.1944 & 0.1412 & 1.1498 \\
\hline (DPnE) & -0.0942 & -0.1655 & 0.1336 & -0.1292 & 0.1442 & 0.9648 \\
\hline
\end{tabular}

تم حساب الثوابت الفيزيائية الأخرى للايثرات مثل مستويات الطاقة الكيميائي (ر) ،المؤشر الاككتروفيلي العالمي، عزم ثنائي القطب (DM) ومستويات طاقة الهومو والومو. (w) نظريًا باستخدام b3lyp/6-311++G** تم عرض هذه النتائج في الجدول 3 حيث يلاحظ انخفاض في قيم جميع الثوابت الفيزياوية مع ازدياد طول السلسة المعوضة على ذرة الاوكسجين نتيجة لانخفاض تاثير الثحنة السالبة للاوكسجين نتيجة الازدياد التدريجي لمجموعة

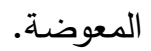
الجدول (3): قيم الثوابت الفيزيائية للايثرات والمحسوبة نظريا باستخدام نظرية *b3lyp/6-311++G

\begin{tabular}{cccccc}
\hline Comp. & HOMO & LUMO & $\boldsymbol{\eta}$ & $\boldsymbol{\mu}$ & $\mathbf{W}$ \\
\hline (DME) & -0.26526 & -0.00832 & 0.12847 & -0.1368 & 0.072824 \\
(DEE) & -0.26101 & -0.00734 & 0.126835 & -0.1342 & 0.07097 \\
(DPE) & -0.26019 & -0.00847 & 0.12586 & -0.1343 & 0.071685 \\
(DBE) & -0.25954 & -0.00824 & 0.12565 & -0.1339 & 0.071335 \\
(DPnE) & -0.25925 & -0.00870 & 0.125275 & -0.134 & 0.07164 \\
\hline
\end{tabular}

من أجل إيجاد الطريقة التي تئثر بها هذه الثوابت على معدل سرعة التفاعل، أُجري تحليل الاتحدار الخطي, (جدول 4)

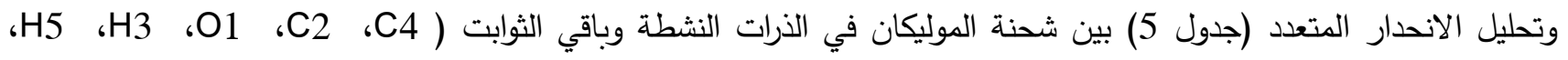




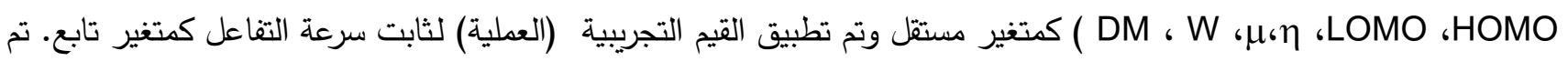
استخراج قيم معامل الارتباط (RE) والخطأ القياسي (SE) والثابت (A) والمتغير (X) من هذه الحسابات. قيمة معامل الارتباط هي المفتاح الرئيس للعثور على العلاقة بين ثابت معدل سرعة التفاعل والمتغيرات الفيزيائية. تم استخدام العوامل الأخرى (SE و A و X لإيجاد القيمة النظرية لثابت معدل السرعة. لذلك ، ستدور المناقثة حول نتائج قيم معامل الارتباط.

ج3lyp/6-311++G** جدول (4): قيم تحليل الانحار الخطي البسيط للثوابت الفيزيائية المحسوبة نظريا باستخذام

\begin{tabular}{ccccccccccccc}
\hline & $\mathbf{0 1}$ & $\mathbf{C 1}$ & H3 & $\mathbf{C 3}$ & H5 & DM & HOMO & LUMO & $\boldsymbol{\eta}$ & $\boldsymbol{\mu}$ & W \\
\hline $\mathbf{R}^{2}$ & $\underline{\mathbf{0 . 9 6 1}}$ & $\underline{\mathbf{0 . 9 4 9}}$ & $\underline{\mathbf{0 . 8 5 7}}$ & 0.655 & 0.193 & 0.252 & $\underline{\mathbf{0 . 9 4 8}}$ & $\underline{\mathbf{0 . 8 0 5}}$ & $\underline{\mathbf{0 . 9 1 4}}$ & $0 . .798$ & 0.616 \\
$\mathbf{S E}$ & $\mathbf{0 . 3 0 7}$ & 0.684 & 3.352 & 0.280 & 9.367 & 2.511 & 24.477 & 2.039 & 18.167 & 103.943 & 43.995 \\
$\mathbf{A}$ & 0.583 & 2.966 & 12.038 & 2.046 & -0.804 & 1.031 & -124.04 & 7.185 & -68.298 & -236.07 & 62.055 \\
$\mathbf{X}$ & -16.463 & 3.379 & -74.76 & -1.523 & 21.962 & 1.267 & -486.63 & 577.889 & 562.106 & -1778.788 & -835.045 \\
\hline
\end{tabular}

تُظهر قيم معاملات الارتباط (R2) في الجدول 4 قيمًا عالية نسبيًا مع ذرات O1 و H3 و C1 في موقع التفاعل وأيضًا لكتغيرات الطاقة (LUMO و HOMO و n) ، انظر القيم الغامقة والمسطرة في الجدول 4. هذه القيم العالية لمعامل الارتباط تعكس العلاقة الجيدة بين ذرات موقع التفاعل والثوابت LUMO و HOMO و n مع ثابت معدل سرعة التفاعل والارتباط المباشر بين هذه الثوابت وثابت سرعة التفاعل. أيضًا ، دعمت العلاقة الجيدة لمتغيرات طاقة الهومو واللومو مع ثابت سرعة التفاعل نظرية أقل طاقة مطلوبة لإزالة الإكترونات من مدار HOMO الخارجي لبدء تفاعل الأكسدة [20]. لذلك، تبدو العلاقة العالية بين قيم المتغيرات ومعدل ثابت السرعة منطقية. بشكل عام ، قد تكون القيم العالية لـ Rاتجة عن قرب تأثير هذه المتغيرات الفيزيائية من موقع تفاعل الايثرات مما يجعل تأثيرها على قيم ثابت السرعة مرتفعًا. وهذا يدل على أن هذه المتغيرات لها تأثير واضح على قيم ثابت السرعة وهذا يتفق مع ما لوحظ في الأدبيات [20].

لدعم النتائج التي استخلصت من تحليل الانحدار البسيط ، تم حساب تحليل الانحدار المتعدد لاستخراج قيم معامل الارتباط (R2)

جدول (5) قيم تحليل الاتحدار الخطي المتعدد للثوابت الفيزيائية المحسوبة نظريا باستخدام b3lyp/6-311++G**

\begin{tabular}{cccccccccccc}
\hline variables & $\mathbf{O 1}$ & $\mathbf{C 2}$ & $\mathbf{H 3}$ & $\mathbf{C 4}$ & $\mathbf{H 5}$ & $\mathbf{D M}$ & HOMO & LUMO & $\boldsymbol{\eta}$ & $\boldsymbol{\mu}$ & $\mathbf{W}$ \\
\hline $\mathbf{O 1}$ & 1 & & & & & & & & & & \\
$\mathbf{C 2}$ & 0.659 & 1 & & & & & & & & & \\
H3 & 0.777 & 0.088 & 1 & & & & & & & & \\
$\mathbf{C 4}$ & 0.825 & $\underline{\mathbf{0 . 9 4 0}}$ & 0.327 & 1 & & & & & & & \\
H5 & 0.423 & 0.868 & 0.007 & 0.688 & 1 & & & & & & \\
DM & 0.458 & 0.830 & 0.186 & 0.733 & 0.454 & 1 & & & & & \\
HOMO & $\underline{\mathbf{0 . 9 9 3}}$ & 0.660 & 0.794 & 0.803 & 0.479 & 0.432 & 1 & & & & \\
LUMO & $\underline{\mathbf{0 . 9 2 0}}$ & 0.812 & 0.539 & $\underline{\mathbf{0 . 9 6 1}}$ & 0.506 & 0.714 & 0.889 & 1 & & & \\
$\boldsymbol{\eta}$ & $\underline{\mathbf{0 . 9 9 0}}$ & 0.744 & 0.707 & 0.893 & 0.504 & 0.565 & $\underline{\mathbf{0 . 9 8 1}}$ & $\underline{\mathbf{0 . 9 6 2}}$ & 1 & & \\
$\boldsymbol{\mu}$ & 0.773 & 0.241 & 0.819 & 0.312 & 0.289 & 0.136 & 0.787 & 0.419 & 0.652 & 1 & \\
$\mathbf{W}$ & 0.763 & 0.814 & 0.309 & $\underline{\mathbf{0 . 9 4 9}}$ & 0.460 & 0.815 & 0.710 & $\underline{\mathbf{0 . 9 5 4}}$ & 0.835 & 0.128 & 1 \\
\hline
\end{tabular}


توضح النتائج الواردة في الجدول 5 اعلاه القيمة العالية لـ R2 المتعلقة بالعلاقة بين متغيرين في نفس الوقت مع معدل ثابت السرعة كما يمكن رؤيته في القيم الغامقة والمسطرة. نلاحظ القيم المهمة بين قيم الثوابت و Rم هي 0.940 و 0.993 و 0.920 و 0.9610 .9810 .990 و 0.9620 .962 و 0.949 و 0.954 على التوالى. يمكن تفسير هذه النتائج نتيجة الارتباط الوثيق بين شحنة O1 و C2 و C4 مما يشير إلى أن شحنات هذه الذرات تتأثر بثدة ببعضها البعض متبوعة بالعلاقة بين هذه الثحنات وقيمة

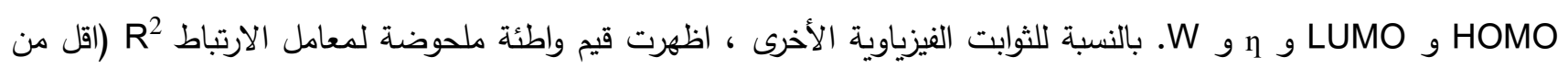
النسبة المقبولة) حيث يمكن استنتاج سبب هذا الانخفاض نتيجة لعدم جود علاقة مؤثرة بين هذه الثوابت وثابت سرعة التفاعل.

كما هو مذكور أعلاه ، تم حساب المعدل التقديري لمعامل سرعة التفاعل للايثرات مع الكلورامين - باستخدام أعلى قيمة كما هو موضح في الجدول 6 اعلاه باستخدام تحليل الانحدار المتعدد. يوضح الجدول 6 قيم معدل ثابت سرعة التفاعل العملي والنظري لتفاعل الايثرات مع الكلورامين -T . عثر على الفروق بين القيم التجريبية والقيم المحسوبة في نطاق حوالي (0.0405) إلى (-0.008). من الواضح أن هذا النطاق المحدود يظهر اتفاقًا جيدًا للغاية بين القيم التجريبية والنظرية لثابت المعدل. أظهرت هذه النتائج تحسنًا في قيمة Rم مقارنة بتحليلات الانحدار الفردي والتي تثير إلى أن معدل تفاعل الأكسدة يعتمد على أكثر من عامل.

جدول (6) قيم ثابت سرعة تفاعل الايثرات مع الكلورامين T العملية والنظية

\begin{tabular}{cccc}
\hline Comp. & $\mathbf{k}_{\exp }$ & $\mathbf{k}_{\text {thio }}$ & $\boldsymbol{\Delta} \mathbf{k}$ \\
\hline 1 & 2.9114 & 2.903349 & -0.0080 \\
2 & 2.6515 & 2.658838 & 0.0073 \\
3 & 2.3704 & 2.393772 & 0.0233 \\
4 & 2.2794 & 2.319952 & 0.0405 \\
5 & 1.8509 & 1.845097 & -0.0058 \\
\hline
\end{tabular}

نتيجة للقيم المتقاربة لثابت سرعة التفاعل المحسوب نظريا مع القيم العملية لثابت التفاعل فان هذه النتائج تدعم ميكانيكية تفاعل أكسدة الايثرات مع الكلورامين - التي بينت ان مركز التفاعل يقع على ذرة الاوكسجين وتتاثر قيمة سرعة التفاعل مع ازدياد طول مجموعة R المعوضة [17.

الاستنتاجات

أظهرت الحسابات Ab-initio والحسابات الإحصائية لتفاعل الايثرات مع الكلورامين-T أن الجزء الأكثر قابلية للتفاعل من جزيء الايثر تجاه الأكسدة هو أصرة O-C وأن الأكسدة تبدأ من ارتباط الكلورامين - على الاوكسجين وأن تفاعل هذه المركبات يعتمد بشكل كبير على الكثافة الإكترونية للاوكسجين،. يمكن ملاحظة دقة هذه الحسابات بوضوح من الاختلافات الطفيفة بين قيم معدل ثابت سرعة السرعة العملي والنظري.

شكر وتقدير

المؤلفون ممتتون لجامعة الموصل وقسم الكيمياء في كلية التربية للعلوم الصرفة على دعمهم وتوفير جميع السبل اللازمة لانجاز لهذا البحث. 


\section{References.}

1. Zhu, R. and M. Lin, Ab initio study of the $\mathrm{ClO}+\mathrm{NH} 2$ reaction: Prediction of the total rate constant and product branching ratios. The Journal of Physical Chemistry A, 2007. 111(19): p. 3977-3983.

2. Raghunath, P. and M. Lin, Ab initio chemical kinetics for the ClOO+NO reaction: Effects of temperature and pressure on product branching formation. The Journal of chemical physics, 2012. 137(1): p. 014315.

3. Xu, Z., R. Zhu, and M. Lin, Ab Initio Studies of ClO $x$ Reactions: VI. Theoretical Prediction of Total Rate Constant and Product Branching Probabilities for the HO2+ClO Reaction. The Journal of Physical Chemistry A, 2003. 107(19): p. 3841-3850.

4. Xu dagger, K., Z. Xu, and M. Lin, Ab initio kinetic prediction of branching rate constants for reactions of H atoms with $\mathrm{CH} 3 \mathrm{O}$ and $\mathrm{CH} 2 \mathrm{OH}$. Molecular Physics, 2007. 105(19-22): p. $2763-$ 2776.

5. Zhu, R. and M. Lin, Ab initio studies of ClOx reactions: Prediction of the rate constants of $\mathrm{ClO}+\mathrm{NO} 2$ for the forward and reverse processes. ChemPhysChem, 2005. 6(8): p. 1514-1521.

6. Zhu, R. and M.-C. Lin, Ab initio studies of ClOX reactions: Prediction of the rate constants of $\mathrm{ClO}+\mathrm{NO}$ for the forward and reverse processes. ChemPhysChem, 2004. 5(12): p. 1864-1870.

7. McNaught, A.D. and A. Wilkinson, Compendium of chemical terminology. Vol. 1669. 1997: Blackwell Science Oxford.

8. Hassan, Y. and N. Saeed, Kinetics and Mechanism of Oxidation of Diethyl Ether by Chloramine-T in Acidic Medium. Journal of Chemistry, 2012. 9(2): p. 642-649.

9. Warren, S. and S. Warren, Solutions Manual to Accompany Organic Chemistry: Jonathan Clayden, Nick Greeves, and Stuart Warren. 2013: Oxford University Press.

10. Vektariene, A., G. Vektaris, and J. Svoboda, A theoretical approach to the nucleophilic behavior of benzofused thieno [3, 2-b] furans using DFT and HF based reactivity descriptors. Arkivoc: Online Journal of Organic Chemistry, 2009.

11. Szabo, A. and N.S. Ostlund, Modern quantum chemistry: introduction to advanced electronic structure theory. 2012: Courier Corporation.

12. Isaacs, N., Molecular orbital calculations on organic molecules-I: CNDO solutions for paraffin hydrocarbons and carbonium ions. Tetrahedron, 1969. 25(16): p. 3555-3566.

13. Zhou, Z. and R.G. Parr, Activation hardness: new index for describing the orientation of electrophilic aromatic substitution. Journal of the American Chemical Society, 1990. 112(15): p. 5720-5724.

14. Neese, F., The ORCA program system. Wiley Interdisciplinary Reviews: Computational Molecular Science, 2012. 2(1): p. 73-78.

15. Jensen, F., Introduction to computational chemistry. 2017: John wiley \& sons.

16. Spss, I., IBM SPSS statistics for Windows, version 20.0. New York: IBM Corp, 2011. 440. 
17. Hassan, Y.I. and N.H. Saeed, Kinetic study of chlorination of p-methoxyacetanilide by chloramine-T in hydrochloric acid medium. Oriental Journal of Chemistry, 2010. 26(2): p. 415.

18. Citra, M.J., Estimating the pKa of phenols, carboxylic acids and alcohols from semi-empirical quantum chemical methods. Chemosphere, 1999. 38(1): p. 191-206.

19. Stasyuk, O.A., et al., Theoretical study of electron-attracting ability of the nitro group: classical and reverse substituent effects. Structural Chemistry, 2015. 26(4): p. 905-913.

20. Hollingsworth, C.A., P.G. Seybold, and C.M. Hadad, Substituent effects on the electronic structure and pKa of benzoic acid. International journal of quantum chemistry, 2002. 90(4-5): p. 1396-1403. 